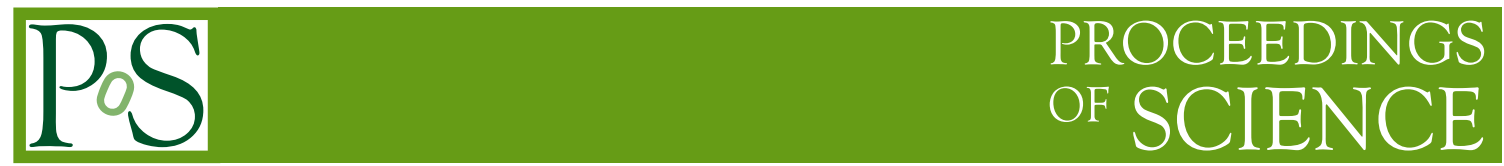

\title{
Radiative Origin of Majorana Neutrino Masses
}

\section{Aristizabal Sierra*}

IFPA, Dep. AGO, Université de Liège, Bat B5, Sart Tilman B-4000 Liège 1, Belgium

E-mail: daristizabaldulg.ac.be

We discuss raditiative-induced neutrino mass mechanisms. We first briefly discuss systematic one-loop level classifications, and then discuss two-loop classification schemes based on a diagrammatic decomposition of the Weinberg operator. We point out that both, the one and two-loop order systematic sorting provides an exhaustive guide for radiative neutrino mass models.

The European Physical Society Conference on High Energy Physics 22-29 July 2015

Vienna, Austria

\footnotetext{
*Speaker.
} 


\section{Introduction}

Results from neutrino oscillation experiments have demonstrated that neutrinos are massive and that the lepton mixing pattern follows a non-trivial structure $[1,2,3]$. These results, combined with the cosmic baryon asymmetry, and cosmological and astrophysical data supporting the hypothesis of dark matter, are of for today the most solid evidence for beyond-the-standard-model (BSM) physics.

Values for neutrino mass square differences are derived from oscillation data [4], while upper limits on the absolute neutrino mass are given by neutrinoless double-beta decay [5], kinematic endpoint measurements of the $\beta$ decay spectrum [6] and cosmology [7]. They all show that neutrino masses are tiny, with values way below the masses of the other standard model (SM) fermions, $\mathscr{O} \sim 0.1 \mathrm{eV}$. Neutrinos, being electrically neutral, are the only SM particles that can have Majorana nature. Testing whether this is the case, requires observing lepton-number-violating (LNV) processes, of which neutrinoless double-beta decay is probably the most promising signature (see e.g. $[8,9]$ for a details discussion). If neutrinos are Majorana particles, their masses will be generated differently compared to the other SM fermions. This, indeed, might be the fundamental reason behind the smallness of neutrino masses.

At the effective level, Majorana neutrino masses can be generated by the dimension five effective operator $\mathscr{O}_{5} \sim \ell \ell H H$, the Weinberg operator [10]. Such a description suffices to account for neutrino oscillation data, however understanding the origin of neutrino masses requires going beyond this effective description and calls for unclosing the nature of the UV completion responsible for this operator.

It is worth pointing out that although a particular realization for $\mathscr{O}_{5}$ (or any higher-order LNV operator) fixes the mechanism for neutrino mass generation, by itself does not provide any information about the origin of the neutrino mixing pattern. The conventional procedure to tackle this problem consist on embedding the model into a larger framework which involves a flavor symmetry. In that context, neutrino mixing is understood as a consequence of flavor symmetry breaking (see refs. [11, 12] for further details). Another interesting approach is that in which the mixing, assumed to be in first approximation of TBM form, results from different mechanisms contributing to the neutrino mass matrix. The main idea is that while one of the mechanisms accounts for the TBM structure, the other one is only responsible for the deviations (see refs. [13]). It is conceivable as well that deviations from the TBM form, and even the origin of CP violation in the lepton sector, arise from deviations from minimality. This can be readily seen e.g. in the type-I seesaw endowed with an $A_{4}$ symmetry [14]. Leading-order terms lead to an exact TBM pattern, additional right-handed neutrinos (and not higher-order effective operators) could-in principle-lead to the experimentally required deviations.

\section{Classification schemes}

Majorana neutrino mass models are realizations of either the Weinberg operator or another higher-order and LNV operator [15]. The number of possibilities is large, and involves UV completions which due to their particle content (or the presence of extra symmetries) generate a neutrino mass matrix either at the tree level or at higher order. For models associated with $\mathscr{O}_{5}$, possibilities 
ranging from tree level up to three-loop level have been considered. Tree level realizations correspond to the standard seesaw models, type-I, II and III. These realizations, not only address the origin of neutrino masses in a natural way, but provide a natural framework for baryogenesis via leptogenesis (see e.g. refs. [16, 17, 18]) and most of it variations [19, 20].

Beyond the tree level, the number of models that have been discussed in the literature is huge (see references in [21, 22] for an almost "complete" list). Examples are the Zee model [23], models with colored scalars [24, 25] and radiative seesaw models [26] ${ }^{1}$. Detailed phenomenological analyses of these realizations have been done, in particular for the Zee and the radiative seesaw models, see e.g. [27, 28]. Recently, one-loop mechanisms within the context of slightly broken lepton number schemes have been discussed as well [32].

For two-loop models one could mention e.g. the Cheng-Li-Babu-Zee model [33, 34, 35], which has been the subject of extensive phenomenological studies (see e.g. [36, 37, 38, 39]). Twoloop snail models, recently pointed out in [40], are as well examples of two-loop neutrino mass generation. At the three-loop order, as an example one could mention the "cocktail" model [41].

Arguably, the main motivation for radiative neutrino mass generation resides on the fact that the loop-suppression factors, combined with extra couplings allows for degrees of freedom whose scale is accessible at high-energy collider, in particular the LHC (see e.g. [42]). However, construction of models based on specific Lagrangians leads to a certain degree of arbitrariness, which-in principle — can be avoided if one relies on the following observation: There is a vast class of models which are just UV completions of $\mathscr{O}_{5}$. Thus, rather than writing particular Lagrangians one could think of starting with a "unique" object and systematically study all its realizations at different orders. Relying on a diagrammatic approach, such a program has been pursued up to the twoloop order [43, 21, 22]. Other attempts aiming at systematic classifications have been presented in $[44,45,46,47,48,49]$.

The diagrammatic approach follows a simple strategy which can be summarized as follows. As soon as the order at which the analysis is going to be done is fixed: (i) find the topologies that can potentially lead to "genuine" diagrams, (ii) from those topologies draw the different diagrams paying special attention to those that can "genuinely" determine the mass matrix, (iii) fix the EW quantum numbers of the BSM fields, (iv) calculate the loop integrals. The list of models resulting from such classification provide a catalog of "genuine" neutrino mass models. With genuine here we refer to diagrams for which the absence of leading-order diagrams can be guaranteed. Only in those cases it can be entirely assured that the neutrino mass matrix originates at the same order that the corresponding diagram.

\section{Brief review of systematic classification of one-loop neutrino mass models}

A diagrammatic-based systematic classification of $\mathscr{O}_{5}$ at the one-loop order has been presented in [21]. In there it was shown that out of the six possible topologies only the one particleirreducibles (1PI) lead to genuine diagrams, shown in fig. 1. It can be noted that with appropriate

\footnotetext{
1 "Hybrid" neutrino mass models where neutrino masses are generated by tree and one-loop contributions can be considered in the type-I seesaw itself, see refs. [29, 30, 31]. Thus, they could be included in the one-loop realizations too.
} 

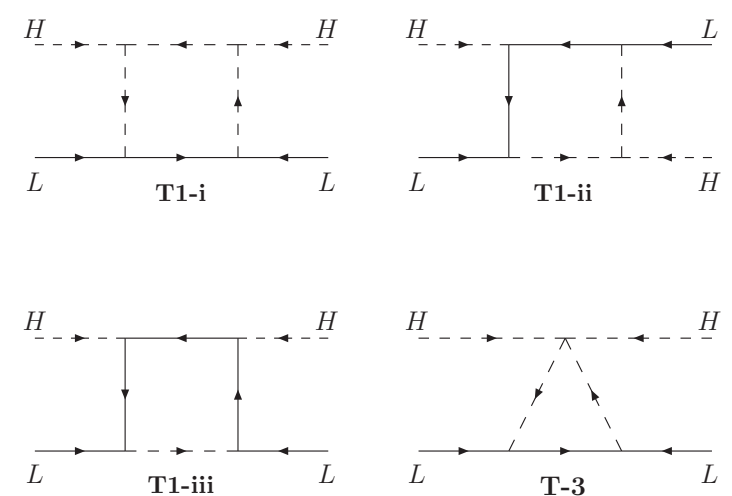

Figure 1: One-loop genuine diagrams according to ref. [21].

quantum number assignments, diagrams T1-ii will lead to the Zee model, while T-3 to the scotogenic model.

A one particle-reducible topology of interest is found in this case. It leads to a set of nongenuine but finite diagrams that can be understood as originating from the type-I, II or III tree level diagrams (depending on the diagram) where one of the vertices (couplings) is one-loop induced. In that sense they can be regarded as "effective" one-loop diagrams. They are of relevance since in those models a small parameter entering in the neutrino mass matrix is justified by its loop-induced nature. EW quantum numbers for the relevant diagrams ${ }^{2}$ as well as explicit expressions for the different one-loop integrals can be found in [21].

\section{Two-loop classification scheme and catalog}

The two-loop diagrammatic analysis has been presented in ref. [22]. In that case the number of topologies is of course larger, something that in turn implies a larger number of diagrams and therefore models. Out of the 29 topologies, only six are found to potentially lead to genuine diagrams, as shown in fig. 2.

Non-genuine but finite diagrams are found as well. Some of them arise from the topologies in fig. 2, but some others from topologies which we here are not listing (for the full list see ref. [22]). As in the one-loop case, they are of interest as well since enable understanding the smallness of small couplings entering in the mass matrix.

To guarantee that the diagrams one gets from the topologies in fig. 2 are truly genuine, additional model-construction rules have to be used, namely:

(I) The resulting particle content should not include hypercharge zero fermion EW singlets and triplets or hypercharge two scalar EW triplets.

(II) The resulting particle content should not contain either hypercharge zero scalar $S U(2)$ singlets or triplets.

\footnotetext{
${ }^{2}$ In the one-loop case the hypercharge of the BSM fields is fixed up to an arbitrary constant, $\alpha$.
} 


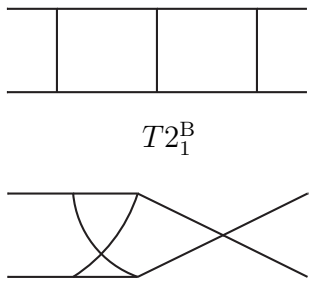

$T 2_{1}^{\mathrm{T}}$

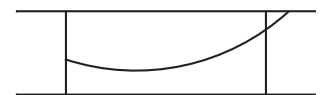

$T 2_{2}^{\mathrm{B}}$

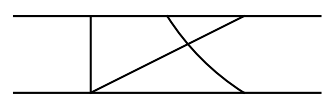

$T 2_{2}^{\mathrm{T}}$
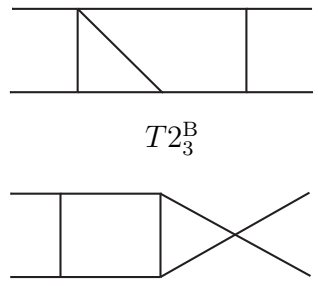

$T 2_{3}^{\mathrm{T}}$

Figure 2: Two-loop topologies that potentially lead to genuine diagrams [22].

(III) Depending on the diagram, BSM scalars should not carry the same quantum numbers than the SM Higgs. In other cases they can, but subject to conditions on the internal Yukawa vertices (see [22] for a detailed discussion).

(IV) For the quartic couplings in diagram T-3 in fig. 1 the following field assignments: $S_{1,2}=S_{D}$, $S_{1}=S_{S}$ and $S_{2}=S_{T}, S_{1}=S_{T}$ and $S_{2}=S_{T}$ (where with $T, D, S$ we refer to EW triplets, doublets and singlets), are possible only if $\left|Y\left(S_{1}\right)-Y\left(S_{2}\right)\right| \neq Y_{H}$, with $Y_{H}$ referring to the Higgs hypercharge (this rule has an exception, see ref. [22]).

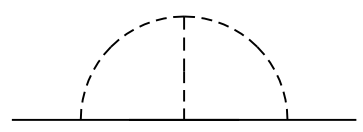

(A)

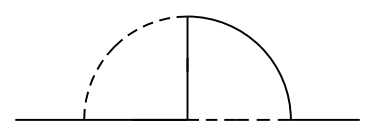

(B)

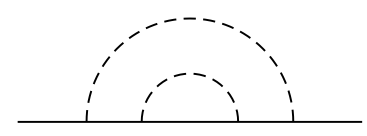

(C)

Figure 3: Different classes of genuine two-loop diagrams. Depending on how the scalar external legs are attached different "models" are obtained.

When complemented with these rules, the topologies in fig. 2 lead to three, and only three, different categories of genuine diagrams, as shown in fig. 3. Depending on how the attachment of the external Higgs lines is done, different diagrams arise. For diagrams in category (A) we have found 10 different possibilities, for (B) six and for (C) four.

With the diagrams at hand, models for two-loop neutrino mass generation can be written provided quantum numbers are specified. For genuine diagrams, this has been given in ref. [22] for EW representations up to triplets. Note that in these models hypercharge for the BSM fields is determined up to two arbitrary constants, $\alpha$ and $\beta$.

The two-loop integrals one can found depend on the chiral structure of internal vertices. However, in the most general case of internal vector-like couplings, the problem involves just four different types of integrals. They were studied long time ago in [50], and were adapted to the different possible models in [22].

\subsection{Using these results to construct two-loop models}

In what follows we illustrate how the two-loop model-building catalog of ref. [22] can be used by means of an example. We believe this is more "illuminating" than just displaying tables with EW quantum numbers. The example is based on a diagram falling in category (B), as shown in fig. 


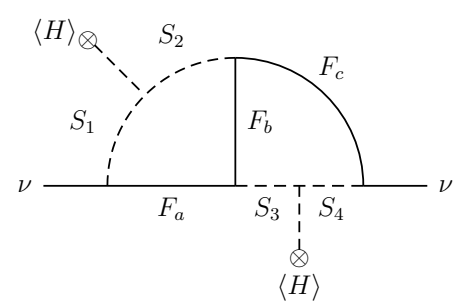

Figure 4: Genuine two-loop model example. The diagram falls into category (B).

\begin{tabular}{|c||c|c|c|c|c|c|c|}
\hline \multicolumn{7}{|c|}{ Category (B) model example } \\
\hline FIELDS & $F_{a}$ & $F_{b}$ & $F_{c}$ & $S_{1}$ & $S_{2}$ & $S_{3}$ & $S_{4}$ \\
\hline$S U(2)_{L}$ & 1 & 2 & 2 & 2 & 1 & 2 & 1 \\
$U(1)_{Y}$ & 1 & 5 & -4 & 2 & 1 & -4 & -3 \\
\hline
\end{tabular}

Table 1: Quantum numbers for the two-loop example.

4. The quantum numbers for the BSM fields were taken from the tab. 3 in [22], and are displayed in tab. 1. They correspond to the hypercharge constants choice $\alpha=2$ and $\beta=-3$.

The diagram in fig. 4 follows from the Lagrangian

$$
\mathscr{L}_{\text {int }}=Y_{i a}\left(\overline{L_{i}^{c}} P_{L} S_{1}\right) \cdot F_{a}^{c}+Y_{c j}\left(\overline{F_{c}} P_{L} L_{j}\right) \cdot S_{4}+h_{a b} \overline{F_{a}^{c}} \cdot\left(F_{b}^{c} S_{3}^{\dagger}\right)+h_{b c}\left(\overline{F_{b}^{c}} F_{c}\right) \cdot S_{2}^{\dagger}+\text { H.c. },
$$

and the scalar potential:

$$
V \supset \mu_{34} S_{4}^{\dagger} \cdot\left(S_{3} H\right)+\mu_{12} S_{2} \cdot\left(S_{1}^{\dagger} H\right)+\text { H.c. }+\sum_{x=1}^{4} m_{S_{x}}^{2}\left|S_{x}\right|^{2}
$$

where the parenthesis refer to $S U(2)$ contractions. The fermions, being vector-like, allow for the following terms

$$
\mathscr{L}_{M}=\sum_{A=a, b, c} m_{F_{A}} \overline{F_{A}} F_{A} .
$$

Coupling $\mu_{34}$ in (4.2) implies mixing between the $Q=3 / 2$ scalars, while $\mu_{12}$ between the $Q=1 / 2$ states. The mass matrices can then be written as

$$
M_{S^{Q=3 / 2}}^{2}=\left(\begin{array}{cc}
m_{S_{3}}^{2} & \mu_{34} v \\
\mu_{34} v & m_{S_{4}}^{2}
\end{array}\right), \quad M_{S^{Q=1 / 2}}^{2}=\left(\begin{array}{cc}
m_{S_{1}}^{2} & \mu_{12} v \\
\mu_{12} v & m_{S_{2}}^{2}
\end{array}\right) .
$$

These matrices can be diagonalized with $2 \times 2$ rotation matrices, namely

$$
R_{Q}=\left(\begin{array}{cc}
\cos \theta_{Q} & \sin \theta_{Q} \\
-\sin \theta_{Q} & \cos \theta_{Q}
\end{array}\right)
$$

with the rotation angles written as

$$
\tan 2 \theta_{Q=3 / 2}=\frac{2 \mu_{34} v}{m_{S_{3}}^{2}-m_{S_{4}}^{2}}, \quad \tan 2 \theta_{Q=1 / 2}=\frac{2 \mu_{12} v}{m_{S_{1}}^{2}-m_{S_{2}}^{2}},
$$


where $v \equiv\langle H\rangle$. The full neutrino mass matrix is calculated after rotation of the interactions in (4.1) and (4.2) to the scalar mass eigenstate basis.

The chiral structure of the external vertices for the diagram in fig. 4 is fixed by the SM to be $P_{L}=\left(1-\gamma_{5}\right) / 2$. Since the BSM fermions are vector-like, the internal vertices of the diagram are not chiral. Bearing that in mind, one can then write the neutrino mass matrix as follows:

$$
\mathscr{M}_{v}=\frac{1}{4\left(16 \pi^{2}\right)^{2}}\left(Y_{i a} Y_{c j}+Y_{j a} Y_{c i}\right) h_{a b} h_{b c} \sin 2 \theta_{Q=3 / 2} \sin 2 \theta_{Q=1 / 2} \sum_{A=1}^{4} \sum_{\alpha, \beta}(-1)^{\alpha}(-1)^{\beta} F_{a c, \alpha \beta, b}^{(A)},
$$

where the dimensionful functions $F_{a c, \alpha \beta, b}^{(A)}$ are determined by four types of two-loop integrals:

$$
\begin{aligned}
& F_{a b, \alpha \beta, b}^{(1)}=\frac{m_{F_{a}} m_{F_{c}}}{m_{F_{b}}} \times \pi^{-4} \hat{\mathscr{I}}_{a c, \alpha \beta}, \\
& F_{a b, \alpha \beta, b}^{(2)}=\left(m_{F_{a}}+m_{F_{b}}+m_{F_{c}}\right) \times \pi^{-4} \hat{\mathscr{I}}_{a c, \alpha \beta}^{\left[(k+q)^{2}\right]}, \\
& F_{a c, \alpha \beta, b}^{(3)}=-\left(m_{F_{a}}+m_{F_{b}}\right) \times \pi^{-4} \hat{\mathscr{I}}_{a c, \alpha \beta}^{\left(k^{2}\right)}, \\
& F_{a c, \alpha \beta, b}^{(4)}=-\left(m_{F_{b}}+m_{F_{c}}\right) \times \pi^{-4} \hat{\mathscr{I}}_{a c, \alpha \beta}^{\left(q^{2}\right)} .
\end{aligned}
$$

Rather than given explicit expressions for the integrals $\hat{\mathscr{I}}$, for which the reader is referred to [22], we display their effect in the neutrino mass matrix as a function of the internal fermion mass $F_{b}$ in fig. 5. Note that in this result we have not consider any flavor structure and we have fixed all the Yukawa couplings to 1. In that sense, these results are just representative of the typical overall neutrino mass and have nothing to do with any possible prediction of this model.

The plots were done for scalar mass parameters $m_{S_{1}}^{2}=100^{2} \mathrm{GeV}^{2}$ and $m_{S_{2}}^{2}=m_{S_{1}}^{2}+\Delta m^{2}$ (with $\left.\Delta m^{2}=\mu v\right)$ and fixed $\Delta m^{2}=24.6 \mathrm{GeV}^{2}$ and two different values of $m_{F}=m_{F_{a}}=m_{F_{c}}$ : to the left 1 $\mathrm{GeV}$ and to the right $100 \mathrm{GeV}$. The black curve shows the full $m_{v}$, the other curves show, instead, the different contributions $m_{v}^{(i)}, i=1,2,3$ individually (determined by the functions in (4.8)-(4.10) and the common global factor in (4.7)). We have found that $m_{v}^{(4)}$ is numerically equal to $m_{v}^{(3)}$, while $m_{v}^{(2)}<0$ and we plot the absolute value. Usually the contribution from $m_{v}^{(2)}-m_{v}^{(3)}$ dominates the neutrino mass for small and moderate values of $m_{F_{b}}$, but at large values of $m_{F_{b}}, m_{v}^{(2)}$ and $m_{v}^{(3)}+m_{v}^{(4)}$ tend to cancel each other, such that the only remaining contribution comes from $m_{v}^{(1)}$. In the plots there are some points for $m_{F_{b}}$, for which the different contributions can actually exactly cancel each other. Note also, that for $m_{F_{b}} \rightarrow \infty, m_{v}$ goes to zero, as expected. Obviously, as these plots demonstrate, neutrino masses of the correct order of magnitude can be achieved for a wide range of input parameters.

\section{Conclusions}

After briefly reviewing the systematic classification of the Weinberg operator at the one-loop order, we discussed a general classification for the two-loop case. The method that we have presented, relies on the different topologies and diagrams that can be derived at this level. We have shown that relevant diagrams can be sorted in three, and only three, categories. Thus, complemented with the SM quantum numbers of the new BSM fields they provide a catalog for neutrino mass models at the two-loop level. 

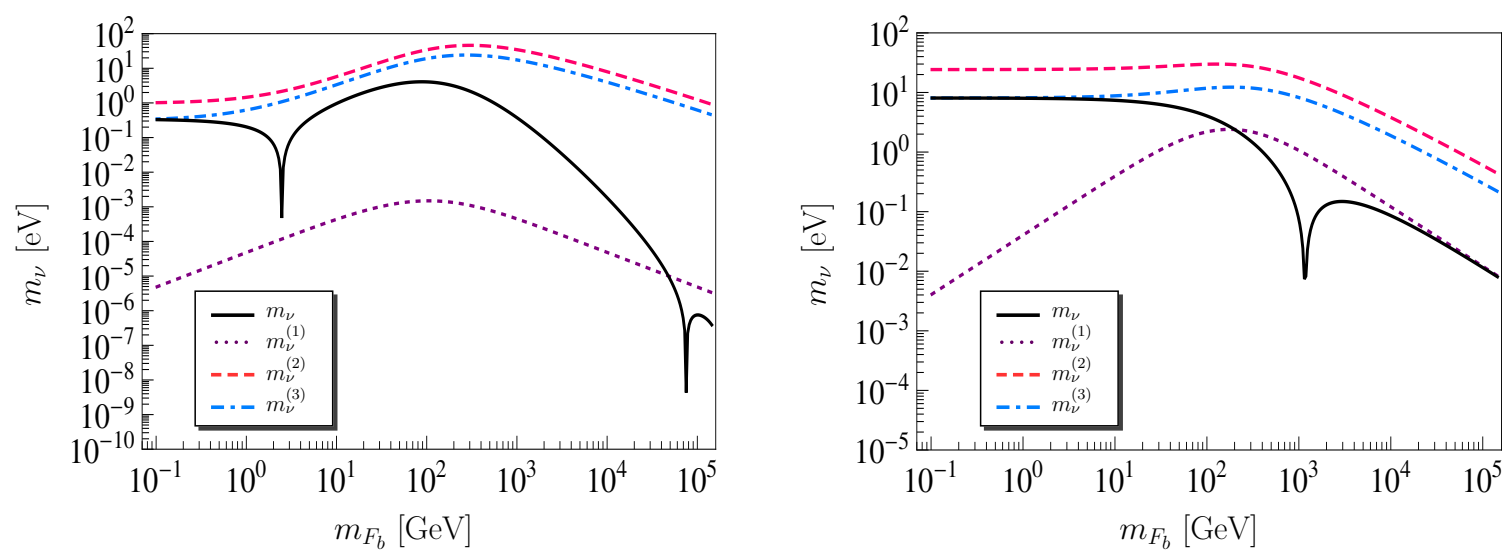

Figure 5: Behavior of the different two-loop integrals at the neutrino mass matrix level. The solid black curve shows the total $m_{v}$, the other curves the individual contributions $m_{v}^{(i)}$, determined by the functions $F_{a b, \alpha \beta, b}^{(i)}$ (see the text). These plots aim only at showing the behavior of the different integrals. They do not represent any phenomenological analysis, both the overall neutrino scale and the values of $m_{F_{b}}$ have to be understood in that way.

Finally, calculation of the corresponding neutrino mass matrices involve at most four different types of two-loop integrals. We have studied their behavior in a simple model, which serves as well to illustrate how the results of the catalog can be used for two-loop neutrino mass model building.

\section{Acknowledgments}

I would like to thank my collaborators Audrey Degee, Luis Dorame and Martin Hirsch. Specially to Martin Hirsch, whose always enlightening observations have led to some of the papers quoted here. I acknowledge financial support from the Belgian FNRS agency through a "Chargé de Recherche" contract.

\section{References}

[1] Y. Fukuda et al. [Super-Kamiokande Collaboration], Phys. Rev. Lett. 81, 1562 (1998) [hep-ex/9807003].

[2] Q. R. Ahmad et al. [SNO Collaboration], Phys. Rev. Lett. 89, 011301 (2002) [nucl-ex/0204008].

[3] K. Eguchi et al. [KamLAND Collaboration], Phys. Rev. Lett. 90, 021802 (2003) [hep-ex/0212021].

[4] D. V. Forero, M. Tortola and J. W. F. Valle, Phys. Rev. D 90, no. 9, 093006 (2014) [arXiv:1405.7540 [hep-ph]].

[5] M. Auger et al. [EXO Collaboration], Phys. Rev. Lett. 109, 032505 (2012) [arXiv:1205.5608 [hep-ex]].

[6] C. Kraus et al., Eur. Phys. J. C 40, 447 (2005) [hep-ex/0412056].

[7] P. A. R. Ade et al. [Planck Collaboration], arXiv:1502.01589 [astro-ph.CO].

[8] W. Rodejohann, J. Phys. G 39, 124008 (2012) [arXiv:1206.2560 [hep-ph]].

[9] F. F. Deppisch, M. Hirsch and H. Pas, J. Phys. G 39, 124007 (2012) [arXiv:1208.0727 [hep-ph]]. 
[10] S. Weinberg, Phys. Rev. D 22, 1694 (1980).

[11] S. F. King, arXiv:1510.02091 [hep-ph].

[12] S. Morisi and J. W. F. Valle, Fortsch. Phys. 61, 466 (2013) [arXiv:1206.6678 [hep-ph]].

[13] D. Aristizabal Sierra, I. de Medeiros Varzielas and E. Houet, Phys. Rev. D 87, no. 9, 093009 (2013) [arXiv:1302.6499 [hep-ph]]; D. Aristizabal Sierra and I. de Medeiros Varzielas, JHEP 1407, 042 (2014) [arXiv:1404.2529 [hep-ph]]; D. Aristizabal Sierra, F. Bazzocchi and I. de Medeiros Varzielas, Nucl. Phys. B 858, 196 (2012) [arXiv:1112.1843 [hep-ph]]; D. Aristizabal Sierra, M. Hirsch, J. W. F. Valle and A. Villanova del Moral, Phys. Rev. D 68, 033006 (2003) [hep-ph/0304141].

[14] G. Altarelli and F. Feruglio, Nucl. Phys. B 741, 215 (2006) [hep-ph/0512103].

[15] F. Bonnet, D. Hernandez, T. Ota and W. Winter, JHEP 0910, 076 (2009) [arXiv:0907.3143 [hep-ph]].

[16] S. Davidson, E. Nardi and Y. Nir, Phys. Rept. 466, 105 (2008) [arXiv:0802.2962 [hep-ph]].

[17] D. Aristizabal Sierra, M. Dhen and T. Hambye, JCAP 1408, 003 (2014) [arXiv:1401.4347 [hep-ph]].

[18] D. Aristizabal Sierra, J. F. Kamenik and M. Nemevsek, JHEP 1010, 036 (2010) [arXiv:1007.1907 [hep-ph]].

[19] D. Aristizabal Sierra, L. A. Munoz and E. Nardi, Phys. Rev. D 80, 016007 (2009) [arXiv:0904.3043 [hep-ph]].

[20] D. Aristizabal Sierra, M. Losada and E. Nardi, Phys. Lett. B 659, 328 (2008) [arXiv:0705.1489 [hep-ph]].

[21] F. Bonnet, M. Hirsch, T. Ota and W. Winter, JHEP 1207, 153 (2012) [arXiv:1204.5862 [hep-ph]].

[22] D. Aristizabal Sierra, A. Degee, L. Dorame and M. Hirsch, JHEP 1503, 040 (2015) [arXiv:1411.7038 [hep-ph]].

[23] A. Zee, Phys. Lett. B 93, 389 (1980) [Phys. Lett. B 95, 461 (1980)].

[24] D. Aristizabal Sierra, M. Hirsch and S. G. Kovalenko, Phys. Rev. D 77, 055011 (2008) [arXiv:0710.5699 [hep-ph]].

[25] P. Fileviez Perez and M. B. Wise, Phys. Rev. D 80, 053006 (2009) [arXiv:0906.2950 [hep-ph]].

[26] E. Ma, Phys. Rev. D 73, 077301 (2006) [hep-ph/0601225].

[27] D. Aristizabal Sierra and D. Restrepo, JHEP 0608, 036 (2006) [hep-ph/0604012].

[28] D. Aristizabal Sierra, J. Kubo, D. Restrepo, D. Suematsu and O. Zapata, Phys. Rev. D 79, 013011 (2009) [arXiv:0808.3340 [hep-ph]].

[29] W. Grimus and H. Neufeld, Nucl. Phys. B 325, 18 (1989).

[30] A. Pilaftsis, Z. Phys. C 55, 275 (1992) [hep-ph/9901206].

[31] D. Aristizabal Sierra and C. E. Yaguna, JHEP 1108, 013 (2011) [arXiv:1106.3587 [hep-ph]].

[32] P. S. B. Dev and A. Pilaftsis, Phys. Rev. D 86, 113001 (2012) [arXiv:1209.4051 [hep-ph]].

[33] T. P. Cheng and L. F. Li, Phys. Rev. D 22, 2860 (1980).

[34] A. Zee, Nucl. Phys. B 264, 99 (1986).

[35] K. S. Babu, Phys. Lett. B 203, 132 (1988).

[36] D. Aristizabal Sierra and M. Hirsch, JHEP 0612, 052 (2006) [hep-ph/0609307]. 
[37] M. Nebot, J. F. Oliver, D. Palao and A. Santamaria, Phys. Rev. D 77, 093013 (2008) [arXiv:0711.0483 [hep-ph]].

[38] J. Herrero-Garcia, M. Nebot, N. Rius and A. Santamaria, Nucl. Phys. B 885, 542 (2014) [arXiv:1402.4491 [hep-ph]].

[39] D. Schmidt, T. Schwetz and H. Zhang, Nucl. Phys. B 885, 524 (2014) [arXiv:1402.2251 [hep-ph]].

[40] Y. Farzan, JHEP 1505, 029 (2015) [arXiv:1412.6283 [hep-ph]].

[41] M. Gustafsson, J. M. No and M. A. Rivera, Phys. Rev. Lett. 110, no. 21, 211802 (2013) [Phys. Rev. Lett. 112, no. 25, 259902 (2014)] [arXiv:1212.4806 [hep-ph]].

[42] S. M. Boucenna, S. Morisi and J. W. F. Valle, Adv. High Energy Phys. 2014, 831598 (2014) [arXiv:1404.3751 [hep-ph]].

[43] E. Ma, Phys. Rev. Lett. 81, 1171 (1998) [hep-ph/9805219].

[44] K. S. Babu and C. N. Leung, Nucl. Phys. B 619, 667 (2001) [hep-ph/0106054].

[45] K. w. Choi, K. S. Jeong and W. Y. Song, Phys. Rev. D 66, 093007 (2002) [hep-ph/0207180].

[46] A. de Gouvea and J. Jenkins, Phys. Rev. D 77, 013008 (2008) [arXiv:0708.1344 [hep-ph]].

[47] F. del Aguila, A. Aparici, S. Bhattacharya, A. Santamaria and J. Wudka, JHEP 1206 (2012) 146 [arXiv:1204.5986 [hep-ph]].

[48] P. W. Angel, N. L. Rodd and R. R. Volkas, Phys. Rev. D 87, no. 7, 073007 (2013) [arXiv:1212.6111 [hep-ph]].

[49] Y. Farzan, S. Pascoli and M. A. Schmidt, JHEP 1303, 107 (2013) [arXiv:1208.2732 [hep-ph]].

[50] J. van der Bij and M. J. G. Veltman, Nucl. Phys. B 231, 205 (1984). 